UDC 517.9

\title{
NONMONOTONICITY OF KNEADING INVARIANTS IN THE FAMILY OF KINKED MAPS ${ }^{*}$ \\ НЕМОНОТОННІСТЬ НІДІНГ ІНВАРІАНТІВ ДЛЯ СІМ'Ї КУСКОВО-ЛІНІЙНИХ ВІДОБРАЖЕНЬ
}

\section{O. Yu. Volkova}

\author{
Inst. Math. Nat. Acad. Sci. Ukraine \\ Tereshchenkivs'ka Str., 3, Kyiv, 01601, Ukraine \\ e-mail: oxana@imath.kiev.ua
}

\begin{abstract}
We study monotonicity properties of the kneading invariant for one-parameter families of piecewise linear unimodal maps, and prove a theorem on violation of monotonicity of the kneading invariant for maps that are symmetric, convex, and consist of four linear pieces. The fact that such maps can not be approximated with smooth mappings that have negative Schwarzian is proved using a dynamics argument.

Досліджуються властивості монотонності нідинг інваріанту для однопараметричних сімей кусково-лінійних унімодальних відображень. Доведено теорему про порушення монотонності нідинг інваріанту для відображень, які симетричні, опуклі та складаються з чотирьох лінійних кусків. Наведено динамічне доведення факту, що такі відображення не можна апроксимувати гладкими відображеннями з від'ємним шварціаном.
\end{abstract}

1. Introduction. Let $f_{a}:[0,1] \rightarrow[0,1], a \in\left[a_{1}, a_{2}\right]$, be a family of maps such that $f_{a}=a \cdot f$, where $f$ is a unimodal map with a unique critical point $c$, i.e., a continuous map that is strictly increasing on the left of $c$ and strictly decreasing on the right of $c$. We assume that $f(0)=$ $=f(1)=0$.

The kneading invariant of $f_{a}$ is an infinite sequence $K=e_{1} e_{2} e_{3} \ldots$ of symbols $0, C$, and 1 defined by

$$
e_{k}= \begin{cases}1 & \text { if } f_{a}^{k}(c)>c \\ C & \text { if } f_{a}^{k}(c)=c \\ 0 & \text { if } f_{a}^{k}(c)<c\end{cases}
$$

For sequences of symbols $0, C, 1$, there is a signed lexicographical order $\prec$ defined in the following way: if $K$ and $K^{\prime}$ coincide up to entry $n-1$ and $e_{n} \prec e_{n}^{\prime}$ (where $0 \prec C \prec 1$ ), then $K \prec K^{\prime}\left(K \succ K^{\prime}\right)$ if the number of the symbol 1 in $e_{1} \ldots e_{n-1}$ is even (odd).

We are interested in the problem:

$$
\text { Is } K\left(f_{a}\right) \text { a monotone function of } a \text { ? }
$$

The same question is asked for topological entropy $h_{\text {top }}$ of $f_{a}$, i.e.: is $h_{\text {top }}\left(f_{a}\right)$ a monotone function of $a$ ?

It is well-known that the topological entropy of a unimodal map depends only on its kneading invariant and the dependence is monotone with respect to the signed lexicographical order

* The research was supported by a visitors grant of the London Mathematical Society.

(C) O. Yu. Volkova, 2005

ISSN 1562-3076. Нелінійні коливання, 2005, m. 8, N으 
indicated above (see [1]). Hence, monotonicity of kneading invariant implies monotonicity of topological entropy.

The problem (1) concerns the phenomenon that unimodal maps become more complicated topologically if the map increases. This phenomenon was observed in many systems in physics and biology (see e.g. [2,3]). But in general (1) is not true. A positive answer is known only for few families of unimodal maps. In particular, nondecreasing of kneading invariant was proved for several polynomial families, $f_{a}(x)=a x(1-x)[1,4,5], f_{a}(x)=a-x^{l}$, for $l=4,6,8, \ldots$ [6] and $f_{a}(x)=a x^{2}(1-x)$ [4]. Proofs for these families use methods of complex analysis and no "real"proof is known. Note that the maps from the families indicated above have negative Schwarzian derivative. A positive answer to the problem (1) is known for several families of piecewise linear maps $[4,5,7]$. In particular, for the symmetric family of piecewise linear maps with 2 pieces (so called tent maps) the kneading invariant is strictly increasing with respect to the parameter. Note that the piecewise linear maps have zero Schwarzian derivative. However, several counter examples have been found. The first example was given by Zdunik [8]. She constructed piecewise-linear maps which consist of 8 linear pieces, convex, symmetric and cannot be approximated in $C^{0}$ topology by maps with negative Schwarzian derivative. Other examples are due to Nusse and Yorke [9] and Kolyada [10], but the map which they constructed is not convex. Other examples can be found in [11]. Due to these examples, for the family of maps $f_{a}=a \cdot f$ the standing conjecture is:

Conjecture 1. If $f_{a}=a \cdot f$ is a family of convex unimodal maps with negative Schwarzian derivative then $K\left(f_{a}\right)$ is a nondecreasing function of $a$.

In this paper we improve on Zdunik's result in the sense that we show that the kneading invariant can be nonmonotone for unimodal maps which are convex, symmetric, but consist of 4 linear pieces. We call such maps one-kinked maps (for precise definition see Section 2). We should point out that nonmonotonicity of kneading invariant among (one)-kinked maps was known. For example, in [12] (Chapter 9) it was mentioned that Bielefeld found a counter example to monotonicity of the kneading invariant for some families of kinked maps. However, to our knowledge, no explanation why this is true was ever published. In this paper we show the mechanism why nonmonotonicity takes place in the families of kinked maps. Furthermore, we construct an open region of kinks for which monotonicity fails.

In addition, we give a fundamental reason why our scheme of getting nonmonotonicity of kneading invariants is not possible for maps with negative Schwarzian derivative.

2. Main result. A family of unimodal maps $f_{a}$ is called a family of one-kinked maps if $f_{a}=$ $=a \cdot f_{A}$, where $f_{A}$ is convex and consists of 4 linear pieces, see [12] (Chapter 9). We assume that $f_{A}(0)=f_{A}(1)=0, f_{A}(c)=1$, where $c=1 / 2$ is the unique critical point of $f_{A}$ and $f_{A}$ is symmetric. The kink $A=\left(x_{1}, y_{1}\right) \in(0,1 / 2) \times(0,1)$ is the point of the graph of $f_{A}$ to the left of the critical point such that $f_{A}$ is not differentiable (Fig. 1).

We write the family of one-kinked maps $f_{a}:[0,1] \rightarrow[0,1], a \in[0,1]$ as

$$
f_{a}(x)= \begin{cases}s_{1}(a) x & \text { if } 0 \leq x \leq x_{1} \\ s_{2}(a)(x-c)+a & \text { if } x_{1}<x \leq c \\ s_{2}(a)(c-x)+a & \text { if } c<x \leq 1-x_{1} \\ s_{1}(a)(1-x) & \text { if } 1-x_{1}<x \leq 1\end{cases}
$$




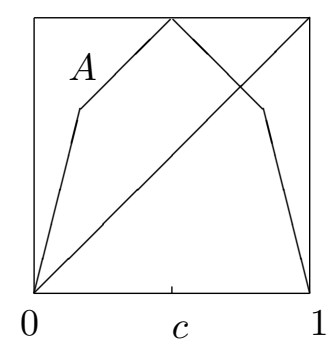

Fig. 1. The map $f_{A}$.

where $s_{1}(a)=a \frac{y_{1}}{x_{1}}$ and $s_{2}(a)=a \frac{2\left(1-y_{1}\right)}{1-2 x_{1}}$. Also we will denote $c_{n}(a)=f_{a}^{n}(c)$. We prove the following theorem:

Theorem 1. If the one-kinked family of maps $f_{a}$ has the following properties:

1) $a_{*}$ is such that $\left|f_{a_{*}}^{\prime}\left(q_{a_{*}}\right)\right|=1$, where $q_{a_{*}}$ the is the orientation reversing fixed point of $f_{a_{*}}$;

2) $c_{2}\left(a_{*}\right)<c<q_{a_{*}}<c_{3}\left(a_{*}\right)<1-x_{1}$.

Then there exists $a_{0}$ such that for all $a \in\left(a_{*}, a_{0}\right) K\left(f_{a}\right) \prec K\left(f_{a_{*}}\right)$.

Proof. To prove the theorem we show how the kneading invariant of the one-kinked map $K\left(f_{a}\right)$ changes when we change the parameter $a$.

$a_{1}<1 / 2$ is close to $1 / 2$ then $c_{1}\left(a_{1}\right)=a_{1}$, the slope $s_{2}\left(a_{1}\right)$ of the map $f_{a_{1}}$ is less than 1 and there exists a fixed point $x_{1}<q_{a_{1}}<1 / 2$ (i.e., $\left.f_{a_{1}}\left(q_{a_{1}}\right)=q_{a_{1}}\right)$ which is attracting. Therefore $K\left(f_{a_{1}}\right)=0^{\infty}$ (Fig. 2).

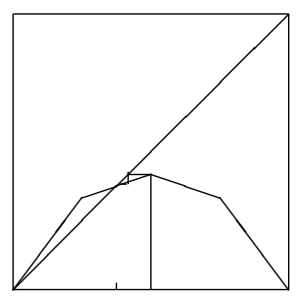

$$
q_{a_{1}} c
$$

Fig. 2. The map $f_{a_{1}}$.

$a_{2}=1 / 2$, then $c_{1}\left(a_{2}\right)=a_{2}=1 / 2$ and there exists a fixed point $q_{a_{2}}=1 / 2$ and $K\left(f_{a_{2}}\right)=$ $=C^{\infty}$ (Fig. 3).

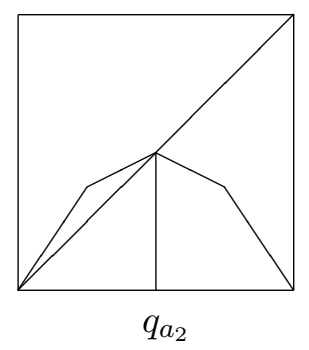

Fig. 3. The map $f_{a_{2}}$. 
$a_{3}>a_{2}$ is close to $a_{2}$ such that $1 / 2<c_{1}\left(a_{3}\right)=a_{3}<1-x_{1}$, the slope $\left|s_{2}\left(a_{3}\right)\right|<1$ and there exists a fixed point $1 / 2<q_{a_{3}}<1-x_{1}$ which is attracting. Therefore $K\left(f_{a_{3}}\right)=1^{\infty}$ (Fig. 4).

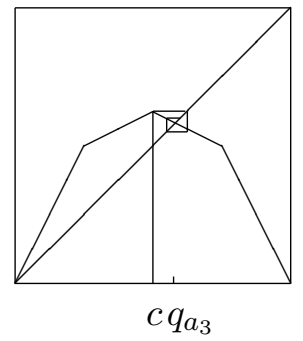

Fig. 4. The map $f_{a_{3}}$.

$a_{4}$ is a bit larger than $a_{3}$ such that $c_{1}\left(a_{4}\right)=a_{4}>1-x_{1}$ and $c_{2}=1 / 2$ (therefore $a_{4}=$ $\left.=1 / 2+\sqrt{1 / 4-x_{1} / 2 y_{1}}\right)$. Then the slope $\left|s_{2}\left(a_{4}\right)\right|<1$, the fixed point $q_{a_{4}}>1 / 2$ is attracting and $K\left(f_{a_{4}}\right)=(1 C)^{\infty}$ (Fig. 5).

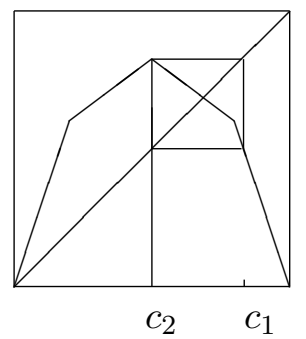

Fig. 5. The map $f_{a_{4}}$.

$a_{4}<a_{5}<a_{*}$ is close to $a_{*}$. Then since by the assumption of the theorem $c_{2}\left(a_{*}\right)<1 / 2<$ $<q_{a_{*}}<c_{3}\left(a_{*}\right)<1-x_{1}$, using continuity argument $c_{2}\left(a_{5}\right)<1 / 2<q_{a_{5}}<c_{3}\left(a_{5}\right)<1-x_{1}$. It follows that $c_{4}\left(a_{5}\right)<q_{a_{5}}$ since $f_{a_{5}}$ is orientation reversing for $x>1 / 2$. Also since $\left|s_{2}\left(a_{5}\right)\right|$ is a bit less than 1, the fixed point $q_{a_{5}}$ is attracting and $c_{4}\left(a_{5}\right)=s_{2}\left(a_{5}\right)\left(1 / 2-c_{3}\left(a_{5}\right)\right)+a_{5}>$ $>c_{1}\left(a_{5}\right)-\left(c_{3}\left(a_{5}\right)-1 / 2\right)>c_{1}\left(a_{5}\right)-\left(1-x_{1}-1 / 2\right)>1 / 2$. I.e., $c_{2}\left(a_{5}\right)<1 / 2<c_{4}\left(a_{5}\right)<q_{a_{5}}<$ $<c_{3}\left(a_{5}\right)<1-x_{1}$. Therefore $K\left(f_{a_{5}}\right)=101^{\infty}$.

$a_{6}=a_{*}$. By the assumptions of the theorem, a fixed point $q_{a_{*}}>1 / 2$ is neutral $\left(\left|f_{a_{*}}^{\prime}\left(q_{a_{*}}\right)\right|=\right.$ $=1)$ and $c_{2}\left(a_{*}\right)<1 / 2<q_{a_{*}}<c_{3}\left(a_{*}\right)<1-x_{1}$. This implies that $1 / 2<c_{4}\left(a_{*}\right)<q_{a_{*}}$, $c_{5}\left(a_{*}\right)=c_{3}\left(a_{*}\right)$ and therefore $K\left(f_{a_{*}}\right)=101^{\infty}$ (Fig. 6).

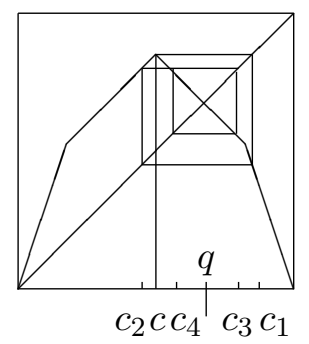

Fig. 6. The map $f_{a_{*}}$. 
$a_{7}>a_{*}$, such that $a_{7}$ is close to $a_{*}$. Then $c_{4}\left(a_{7}\right)$ lies close to $c_{4}\left(a_{*}\right)$ and $q_{a_{7}}$ is close to $q_{a_{*}}$, hence $c_{4}\left(a_{7}\right)<q_{a_{7}}$. So there exists a $k_{0}>2$ such that for $2<k<k_{0}, 1 / 2<c_{2 k}\left(a_{7}\right)<$ $<c_{2(k-1)}\left(a_{7}\right), c_{2 k_{0}-1}\left(a_{7}\right)>1 / 2, c_{2 k_{0}}\left(a_{7}\right)<1 / 2$. This means that the kneading invariant $K\left(f_{a_{7}}\right)=10111 \ldots 10$, where the number of 1 before the second 0 is even. Therefore $K\left(f_{a_{7}}\right)<$ $<K\left(f_{a_{*}}\right)=101^{\infty}$. In addition, $q_{a_{7}}>1 / 2$ is repelling $\left(\left|f_{a_{7}}^{\prime}\left(q_{a_{7}}\right)\right|>1\right)$.

The theorem is proved.

Remark 1. Comparing the family $f_{a}$ to the family of quadratic maps $Q_{a}(x)=a x(1-x)$ we have the following:

if $a<2$ then $K\left(Q_{a}\right)=0^{\infty}$ and $q_{a}<1 / 2$ is attracting;

if $a=2$ then $K\left(Q_{a}\right)=C^{\infty}$ and $q_{a}=1 / 2$;

if $2<a<3$ then $K\left(Q_{a}\right)=1^{\infty}$ and $q_{a}>1 / 2$ is attracting;

if $a=3$ then $K\left(Q_{a}\right)=1^{\infty}$ and $q_{a}>1 / 2$ is neutrally attracting $\left(\left|Q^{\prime}\left(q_{a}\right)\right|=1\right)$;

if $a>3$ then $K\left(Q_{a}\right)=1^{\infty}$ and $q_{a}>1 / 2$ is repelling $\left(\left|Q^{\prime}\left(q_{a}\right)\right|>1\right)$ and there exists a period 2 orbit $1 / 2<Q(p)<q_{a}<p$; this orbit is attracting;

if $a=a_{*}$ then $K\left(Q_{a_{*}}\right)=101^{\infty}$ and $q_{a}=Q_{a}^{3}(1 / 2)$ is repelling and there are no attracting periodic points.

Lemma 1. Let $f_{a}$ be a unimodal map, $c$ its critical point, $S f_{a}<0$ and suppose there exists a nonrepelling periodic orbit $x_{1}, x_{2}, \ldots, x_{n}$. Then one of the $x_{i}$ has $c$ in its immediate basin.

Proof. See [13] (Chapter 1.11).

Corollary 1.For a unimodal map $f_{a}$ with $S f_{a}<0$ and a fixed point $q_{a}>c$ there is no parameter a for which $K\left(f_{a}\right)=101^{\infty}$ and $q_{a}$ is nonrepelling.

Proof. If such a parameter $a$ exists then, since $S\left(f_{a}\right)<0$ and $q_{a}>c$ is nonrepelling by Lemma $1, q_{a}$ will have $c$ in its immediate basin. Therefore all $c_{n}$ must be greater than $c$. But this is impossible since $c_{2}<c$.

Example. Let $f_{a}$ be a family of one-kinked maps given above. Then for the open region

$$
\begin{aligned}
F=\left\{\begin{array}{l}
A=\left(x_{1}, y_{1}\right) \mid 0< \\
x_{1}<1 / 3,
\end{array}\right. & \\
& \frac{2 x_{1}^{2}-2,5 x_{1}-0,25-\sqrt{4 x_{1}^{4}-6 x_{1}^{3}+3,25 x_{1}^{2}-0,75 x_{1}+0,0625}}{2 x_{1}^{2}-x_{1}-1}< \\
& \left.<y_{1}<\frac{1+5 x_{1}-2 x_{1}^{2}+\sqrt{4 x_{1}^{4}-12 x_{1}^{3}+13 x_{1}^{2}-6 x_{1}+1}}{2\left(2-x_{1}\right)}\right\}
\end{aligned}
$$

Theorem 1 holds. The region $F$ corresponds to the conditions $q_{a_{*}}<c_{3}\left(a_{*}\right), c_{2}\left(a_{*}\right)<1 / 2$ and $c_{3}\left(a_{*}\right)<1-x_{1}$ from Theorem 1. The region $F$ is shown in Fig. 7.

Remarks. 2. It is not difficult to see that a similar result holds for a family of maps with more than one kink. Recall that Zdunik considered the case of three-kinked maps.

3. Modifying slightly a result of Taylor [14] one can construct a smooth and convex function $F$ with the same properties as $f_{a_{*}}$. A local geometric argument of Nusse and Yorke [9] shows 
that any smooth map that approximates kinked maps cannot have negative Schwarzian derivative. Lemma 1 and Corollary 1 above give a dynamical argument why this particular procedure of getting nonmonotonicity cannot be realized at all by maps with negative Schwarzian derivative.

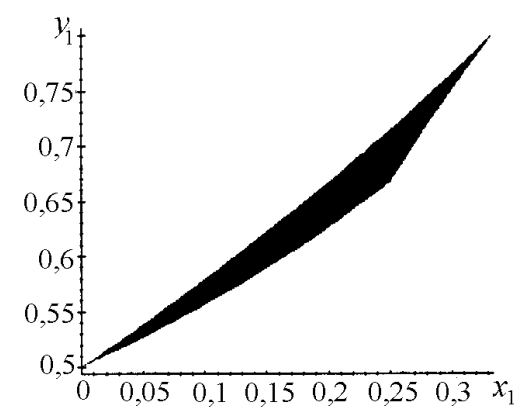

Fig. 7. The region $F$.

1. Milnor J., Thurston W. On iterated maps of the interval // Lect. Notes Math. - 1988. - 1342. - P. $465-563$.

2. Bier M., Bountis T. Remerging Feigenbaum trees in dynamical systems // Phys. Lett. A. - 1984. - 104, № 5. - P. 239-244.

3. Inoue M., Kamifukumoto H. Scenarios leading to chaos in forced Lotka-Volterra model // Progr. Theor. Phys. - 1984. - 71. - P. 930-937.

4. Tsujii M. A simple proof of monotonicity of entropy in the quadratic family using Ruelle operator. - Banach Centre Preprint, 1997.

5. Tsujii M. A simple proof of monotonicity of entropy in the quadratic family // Ergod. Theory and Dynam. Syst. - 2000. - 20. - P. $925-933$.

6. Douady A., Hubbard J. H. Publ. Math. Orsay. - 1984-1985.

7. Brucks K., Misiurewicz M., and Tresser C. Monotonicity properties of the family of trapezoidal maps // Communs Math. Phys. - 1991. - 137. - P. 1-12.

8. Zdunik A. Entropy of transformations of the unit interval // Fund. math. - 1984. - 114. - P. $235-241$.

9. Nusse H., Yorke J. Period halving for $x_{n+1}=M F\left(x_{n}\right)$ where $F$ has negative Schwarzian derivative // Phys. Lett. A. - 1988. - 127. - P. 328-334.

10. Kolyada $S$. One-parameter families represented by integrals with negative Schwarzian derivative violating monotone bifurcations // Ukr. Mat. Zh. - 1989. - 41, № 2. - P. 258-261.

11. Bruin H. Non-monotonicity of entropy of interval maps // Phys. Lett. A. - 1995. - 202. - P. 359-362.

12. Brucks K., Bruin H. Topics from one-dimensional dynamics // London Math. Soc. Student Texts 62. Cambridge Univ. Press, 2004. - 312 p.

13. Devaney $R$. An introduction to chaotic dynamical systems. - California: Benjamin/Cummings Publ. Co., Menlo Park, 1986. - 336 p.

14. Taylor J. A one-parameter family of unimodal, concave, polynomials maps of the interval exhibiting multiplicities // Adv. Appl. Math. - 1991. - 12. - P. 464-481.

Received 25.02.2005 